\title{
Updated methodological guidance for the conduct of scoping reviews.
}

PETERS, M.D.J., MARNIE, C., TRICCO, A.C., POLLOCK, D., MUNN, Z., ALEXANDER, L., MCINERNEY, P., GODFREY, C.M. and KHALIL, $H$. 


\section{Updated methodological guidance for the conduct of scoping reviews}

2 Authors: Micah DJ Peters, ${ }^{1,2,3}$ Casey Marnie, ${ }^{1}$ Andrea C Tricco, ${ }^{4}$ Danielle Pollock,,${ }^{5}$ Zachary Munn, ${ }^{5}$

3 Lyndsay Alexander, ${ }^{6}$ Patricia McInerney, ${ }^{7}$ Christina M Godfrey, ${ }^{8}$ Hanan Khalil, ${ }^{9}$

${ }^{1}$ Rosemary Bryant AO Research Centre, UniSA Clinical and Health Sciences, University of South Australia, Adelaide, Australia.

${ }^{2}$ School of Nursing, Faculty of Health and Medical Sciences, University of Adelaide, Adelaide, Australia.

${ }^{3}$ The Centre for Evidence-based Practice South Australia (CEPSA): a Joanna Briggs Institute Centre of Excellence.

${ }^{4}$ Li Ka Shing Knowledge Institute of St. Michael's Hospital, Unity Health Toronto, Toronto, Canada; Epidemiology Division and Institute of Health Management, Policy, and Evaluation, Dalla Lana School of Public Health University of Toronto, Toronto, Canada; Queen's Collaboration for Health Care Quality Joanna Briggs Institute Centre of Excellence, Queen's University, Kingston, Canada ${ }^{5} \mathrm{JBI}$, Faculty of Health and Medical Sciences, University of Adelaide, Adelaide, Australia ${ }^{6}$ School of Health Sciences, Robert Gordon University, Aberdeen UK. The Scottish Centre for Evidence-based Multi-professional Practice: A Joanna Briggs Institute Centre of Excellence ${ }^{7}$ The Wits-JBI Centre for Evidence-Based Practice, Faculty of Health Sciences, University of the Witwatersrand, Johannesburg, South Africa

${ }^{8}$ Queen's Collaboration for Health Care Quality: A JBI Centre of Excellence, School of Nursing, Queen's University, Kingston, Ontario, Canada ${ }^{9}$ School of Psychology and Public Health. Department of Public Health, La Trobe University, Melbourne, Australia. The Queensland Centre of Evidence Based Nursing and Midwifery, A Joanna Briggs Institute Centre of Excellence.

\section{Corresponding Author:}

Dr Micah DJ Peters

Rosemary Bryant AO Research Centre, University of South Australia

City East Campus, Centenary Building C4-22, North Terrace

Adelaide, South Australia

t: +61 883021423 | e: Micah.Peters@unisa.edu.au

Acknowledgements: We would like to acknowledge the support, feedback, and advice we have received on the development of the updated scoping reviews methodology from the JBI Scientific Committee. We also acknowledge and thank previous members of the group for their past contributions.

Funding: The authors declare no specific funding for this work.

Conflicts of interest: The authors declare no conflicts of interest. 
39 Objective: The objective of this paper is to describe the updated methodological guidance for

40 conducting a JBI scoping review with a focus on new updates to the approach and the development

41 of the Preferred Reporting Items for Systematic Reviews and Meta-Analyses extension for Scoping

42 Reviews (the PRISMA-ScR).

43 Introduction: Scoping reviews are an increasingly common approach to informing decision making 44 and research based on the identification and examination of the literature on a given topic or issue.

45 Scoping reviews draw upon evidence from any research methodology and may also include evidence 46 from non-research sources such as policy. In this manner, scoping reviews provide a comprehensive 47 overview to address typically broader review questions than traditionally more specific systematic reviews of effectiveness or qualitative evidence. The increasing popularity of scoping reviews has been accompanied by the development of a reporting guideline - the PRISMA-ScR. In 2014, the JBI Scoping Review Methodology Group developed guidance for scoping reviews which received minor updates in 2017 and was updated most recently in 2020. The updates reflect ongoing and substantial developments in approaches to scoping review conduct and reporting. As such, the JBI Scoping Review Methodology Group recognized the need to revise the guidance to align it with the current state of knowledge and reporting standards in evidence synthesis.

Methods: Between 2015 and 2020, the JBI Scoping Review Methodology Group expanded its membership, extensively reviewed the literature, engaged via annual face-to-face meetings, regular teleconferences and email correspondence, sought advice from methodological experts, facilitated workshops, and presented at scientific conferences. This process led to updated guidance for scoping reviews published in the JBI Reviewer's Manual. The updated chapter was endorsed by JBI's

60 International Scientific Committee in 2020.

Results: The updated JBI guidance for scoping reviews includes additional guidance on several methodological issues, such as when a scoping review is (or is not) appropriate, and how to extract, 63 analyze, and present results and provides clarification for implications for practice and research. 64 Furthermore, it is aligned with the PRISMA-ScR to ensure consistent reporting.

Conclusions: The latest JBI guidance for scoping reviews provides up to date guidance that can be used by authors when conducting a scoping review. Furthermore, it aligns with the PRISMA-ScR, which can be used to report the conduct of a scoping review. A series of ongoing and future methodological projects identified by the JBI Scoping Review Methodology Group to further refine the methodology are introduced. 


\section{Introduction}

Along with the increased production of primary research, the conduct and publication of evidence syntheses (reviews) has also increased and evolved over time. ${ }^{1}$ The need to synthesize diverse types of evidence underpins the design and evolution of new approaches intended to rigorously identify and synthesize data to answer a range of pressing questions for end users in policy, research, and practice. In 2009, Grant and Booth identified 14 different types of reviews. ${ }^{2}$ By 2016, this variety had increased to 25 evidence synthesis methods, ${ }^{3}$ and 48 review types in $2019 .{ }^{4}$ Scoping reviews, also sometimes referred to as 'mapping reviews' or 'scoping studies' is one approach to evidence synthesis that are increasingly being utilized internationally. $5,6,7,8$ Although it is unclear when the first scoping review was conducted, the first methodological guide for these reviews was published by Arksey and O'Malley in 2005. Arksey \& O'Malley observed and reflected on the early appearance of scoping studies in the literature and noted similarities and a lack of uniformity, and proposed a seminal framework for their conduct. ${ }^{9}$ Arksey and O'Malley also noted the necessity for others to continue their work to further improve guidance for authors to conduct and report scoping reviews. This has occurred over the years and included extensions proposed by Levac and colleagues. ${ }^{10}$ In 2014, the Joanna Briggs Institute (JBI) International Scientific Committee convened a Scoping Review Methodology Group from members of the JBI and the Joanna Briggs Collaboration (JBC). ${ }^{11}$ This group extensively reviewed the literature, engaged via annual face-to-face meetings, regular teleconferences and email correspondence, sought advice from methodological experts, facilitated workshops, and presented at scientific conferences. This process led to the publication of the JBI's first chapter and peer-reviewed paper describing guidance for authors of scoping reviews. ${ }^{12,13}$ Like guidance for the more traditional systematic reviews that the JBI has become known for, the guidance for scoping reviews explicitly addressed the need for scoping reviews to be rigorously conducted, transparent, and trustworthy. The chapter underwent minor updates in $2017,{ }^{14}$ and overall, the JBI guidance has since been used and cited by many review groups around the world from a range of disciplines, academic, and professional backgrounds. ${ }^{15}$ In 2018, the Preferred Reporting Items for Systematic Reviews extension for Scoping Reviews (PRISMA-ScR) was developed by an international team of experts in scoping reviews and evidence synthesis, ${ }^{16}$ including members of the $\mathrm{JBI} / \mathrm{JBC}$ working group, to be consistent with the JBI's scoping review methodology and to provide reviewers with a reporting checklist for their reviews. ${ }^{14}$ This methodological paper provides an overview of scoping review methods and highlights the most recent updates to the JBl's guidance for the conduct of scoping reviews, which was recently published in the JBI Reviewer's Manual. ${ }^{17}$ This updated guidance primarily takes into account the launch of the (PRISMA-ScR), ${ }^{16}$ which is recommended to be used in tandem with the latest JBI guidance. The major areas of update include:

- Inclusion of the PRISMA-ScR reporting guideline and checklist throughout the chapter. 
- Advice on when a scoping review is (or is not) appropriate, and how to extract, analyze, and present results.

- Updates to many of the examples used throughout the chapter and the use of clearer language to remove ambiguity.

- A discussion on the term 'systematic' in relation to scoping reviews and clarifying our preferred terminology for this evidence synthesis approach is 'scoping reviews' (whilst they still remain systematic).

- Updated section on indications for conducting a scoping review.

- Further discussion on the role (or not) of methodological appraisal in scoping reviews.

- Clarification on implications for practice (now called 'implications of the findings').

- Expanded background to the chapter.

Additionally, as interest in the methodology has grown it has come to our attention that in addition to adding new sections, there are also areas throughout the guidance which required clarification, updates, and modification. Some of these changes were informed by feedback from scoping review authors using the guidance (Khalil 2019), ${ }^{15}$ whilst others have been identified by group members themselves or by advances in the methodological literature. It is in light of this evidence that the authors hope an update to the guidance will support improved consistency and rigor in the undertaking and reporting of scoping reviews.

\section{What are scoping reviews and why conduct a scoping review?}

According to the Canadian Institutes of Health Research, scoping reviews are "exploratory projects that systematically map the literature available on a topic, identifying key concepts, theories, sources of evidence and gaps in the research". 18(para 1) Scoping reviews are conducted for several reasons, with the most common being to explore the breadth/depth of the literature, map and summarize the evidence, inform future research, and identify/address knowledge gaps. ${ }^{19}$ Scoping reviews are particularly helpful when the literature is complex and heterogeneous. Scoping reviews can provide useful insight for decision makers into the nature of a concept and how that concept has been studied in the literature over time. They can be used to develop a research agenda, advance the field, and identify areas for future systematic reviews or other types of evidence synthesis. Decision makers in particular find this method of evidence synthesis provides a useful overview of research previously undertaken and reported in the literature, often in regard to the types of programs or interventions that have been examined, informing options for consideration in future research. Indeed, the number of scoping reviews doubled from 2014 until $2017,,^{19}$ demonstrating the popularity of this method in the literature.

\section{Need for scoping reviews to still be systematic}

Initially, JBI's guidance used the terminology 'systematic scoping review'. 12,14,17 This was to signpost the similarities between the JBI's guidance for scoping reviews and the JBI's guidance for other evidence syntheses including systematic reviews that focus on rigor, reproducibility, and 
148 transparency. In this latest update, the nomenclature has been refined to simply 'scoping reviews' in

149 recognition that all types of evidence synthesis should be conducted systematically, as well as to

150 reduce the risk of confusion between different types of review. ${ }^{19,29}$ In addition, 'scoping review' is the

151 most commonly used term to describe a scoping review, so removing the term 'systematic' also

152 improves consistency. ${ }^{19}$ We argue that all types of evidence synthesis should be systematic and

153 follow methodological guidance.

Choosing between a systematic or scoping review approach

156 Given the array of evidence synthesis methodologies and review types, it is critical that authors

157 assess their objectives and intentions prior to the undertaking of any review. This is a particularly

158 pertinent consideration when deciding between a systematic or scoping review as both maintain

159 particular, but separate, value for given aims or outcomes. Broadly speaking, if the intention of the

160 review is to inform clinical decision-making, for example determining the feasibility, appropriateness,

161 meaningfulness or effectiveness of a particular intervention then a systematic review is more

162 appropriate. ${ }^{20}$ Scoping reviews however, are more appropriate in assessing and understanding the

163 extent of the knowledge in an emerging field, or to identify, map, report or discuss the

164 characteristics/concepts in that field. For example, Harfield and colleagues' scoping review identified

165 the characteristics of Indigenous primary health care service delivery models. ${ }^{21}$ Subsequently, they

166 were able to develop and describe a new Indigenous Primary Health Care Service Delivery Model

167 which was able to place importance on the local cultural values, customs and beliefs of Indigenous

168 people. $^{21}$

169

170 The value of scoping reviews to evidence-based healthcare and practice lies in their ability to incorporate various types of literature that are not limited specifically to research studies. For

172 example, scoping reviews can be useful in developing policy maps. Mapping policy documents and

173 research studies has been previously undertaken by Anderson and colleagues in 2008 and Watson

174 and colleagues in 2011.6,22 Both authors used scoping reviews to examine research papers and policy

175 documents to map complex topics.

177 In general, systematic reviews have more focused research questions than scoping reviews, which

178 are much broader. Furthermore, scoping reviews are exploratory and descriptive in nature, whereas

179 systematic reviews, those with meta-analysis or network meta-analysis, can be explanatory or

180 analytical in nature. ${ }^{23}$ An online tool exists that can be used to assist authors when selecting between

181 a systematic review and a scoping review, ${ }^{24}$ by providing general indication of the objective and topic

182 to be reviewed, a user can generate a recommendation towards the most appropriate method of

183 review. Results of scoping reviews can identify further areas for subsequent research and clarify

184 whether a systematic review can be conducted to address a specific question as a consequence of

185 mapping the literature. In general, the indications for scoping reviews can be summarized below: ${ }^{17,20}$

186 - As a precursor to a systematic review.

187 - To identify the types of evidence available in a given field. 
- To identify and analyze knowledge gaps.

- To clarify key concepts/definitions in the literature.

- To examine how research is conducted on a certain topic or field.

- To identify key characteristics or factors related to a concept.

Whilst scoping review methodology has evolved, there is still some confusion of terms with other evidence synthesis approaches such as 'evidence gap maps'. ${ }^{4}$ Evidence gap maps share similarities to scoping reviews in terms of identifying a research question, conducting a systematic search and descriptive analysis, ${ }^{30}$ however, evidence gap maps tend to limit the inclusion of evidence to systematic reviews and primary research studies, and may also include critical appraisal.

\section{Methodological updates}

200 As is characteristic of rigorous evidence synthesis approaches, scoping reviews should be well planned out and driven by a protocol. Protocols are important for pre-definition of the objective, question/s and method and they support transparent and unbiased reporting. The protocol should detail the reviews' inclusion and exclusion criteria and identify what and how data will be extracted and presented. Deviations from the protocol should be clearly highlighted and explained in the ensuing scoping review. Currently, scoping reviews are not able to be registered with the international database of prospectively registered systematic reviews/PROSPERO. However, authors conducting a scoping review should consider publishing, registering or making their protocol available via platforms such as Figshare, Open Science Framework, ResearchGate, ResearchSquare or similar so that it is freely available. The JBI journal JBI Evidence Synthesis is one avenue for publishing scoping review protocols (and their subsequent reviews) that have followed the JBI methodology.

\section{Title and review questions}

213 The title of the protocol and corresponding review should give a clear indication of the topic and

214 identify the manuscript as a scoping review protocol or review. It is also useful to ensure that key

215 elements of the inclusion criteria are reflected in the title to enable easy identification by readers. The

216 'PCC' mnemonic (Population, Concept and Context) is recommended as a guide to construct a clear

217 and meaningful title and inclusion criteria for a scoping review. Use of the PCC mnemonic clearly

218 identifies the focus and context of a review, further enabling utility to the reader. Specific outcomes,

219 interventions or phenomena of interest do not need to be stated for a scoping review, although these

220 details might be helpful for some scoping review topics. There should be congruence between the

221 title, review question/s, and inclusion criteria.

222 A clear scoping review question that incorporates the elements of the PCC guides the development of 223 specific inclusion criteria, facilitates the literature search, and provides a robust structure for the 224 development of the scoping review. A scoping review will generally have one primary question, e.g. 

tonsillectomies with or without adenoidectomies for chronic infections or sleep disordered breathing?"

228 Some scoping reviews may also have one or more sub-questions that delve into particular attributes of Context, Population or Concept. Sub-questions can be useful in outlining how the evidence is likely to be mapped. For example: "What are the ages of the pediatric patients where quality of life questionnaires have been or could be used within the sources of evidence identified for the primary review question?"

233

Inclusion criteria

235 A scoping review's inclusion criteria should be detailed in the protocol and should also provide information regarding the types of sources of evidence that will be considered for inclusion. As scoping reviews are amenable to the inclusion of all methodologies as well as non-research sources such as policy documents or websites the protocol should state which sources will be examined. It is important to note that here, sources of evidence do not refer to the locations of where evidence will be sought, i.e. online databases. These should be stated in the search strategy. The inclusion criteria aid the reader's understanding of the scope of the review and provide a guide for the reviewers themselves to make decisions regarding what sources to include or exclude.

Participants

244 The inclusion criteria should specify important characteristics of the review's participants. This may 245 include age, gender, and other relevant factors appropriate to the review's objective and review 246 question/s. Defining participants per se is not always necessary. For example, a scoping review with 247 the objective of describing the details of research designs used in a specific area of study may not 248 need to detail the types of participants involved in that research.

\section{Concept}

250 The scoping review's main concept/s should be explained. Depending upon the objective and question/s, the 'concept' may include details similar to the elements detailed in a traditional systematic review, such as 'interventions', 'phenomena of interest', or 'outcomes'. For example, the principal concept of interest in the example questions above are quality of life questionnaires used following tonsillectomies. Additional elements of this concept may also be of interest, such as; the format (e.g. paper or web-based), contents (i.e. assessment domains) of the included instruments, and validity and reliability (i.e. if and how they have been psychometrically tested). Outcomes may also be a component of a scoping review's 'concept' and should be linked to the objective review question/s.

258 For example, this scoping review could also identify and map any reported outcomes addressed 259 within quality of life assessments. In other examples, the concept may relate to concepts and 260 definitions (i.e. what definitions have been used to define low-value care') or elements of research 261 design (i.e. methodological details and conduct). 
263 A scoping review's 'context' will vary depending on the objective and question/s and may include

264 details regarding geographic location (e.g. a particular country or region) and/or specific social,

265 cultural, or gender-based factors. Context may also include setting specifics (such as acute care,

266 primary health care, or the community). The context in the example above has not been stated

267 explicitly (i.e. it is 'open') as sources of evidence from any contextual setting would be eligible for

268 inclusion. Specifying the context will aid in refining the scope of the review, such as by focussing only

269 on specific countries or only particular healthcare settings.

\section{Types of evidence sources}

271 A scoping review can include any and all types of literature, e.g. primary research studies, systematic

272 reviews, meta-analyses, letters, guidelines, websites, blogs, etc. Reviewers however may wish to

273 impose limits based on the knowledge that particular types of sources would be most useful and

274 appropriate. The example scoping review above sought certain quantitative studies only; qualitative

275 studies, reviews, and conference abstracts were excluded as these were deemed by the reviewers

276 not to be likely to contain relevant information to answer the review questions.

277

\section{Search strategy}

279 The search strategy for a scoping review should ideally aim to be as comprehensive as possible

280 within the constraints of time and resources in order to identify both published and unpublished (i.e.

281 Gray literature) primary sources of evidence, as well as reviews. Any limitations in terms of the

282 breadth and comprehensiveness of the search strategy should be detailed and justified. A complete

283 search strategy for at least one major database should be included as an appendix to the protocol

284 and in the subsequent review. The input of a research librarian or information scientist can be

285 invaluable in designing and refining the search. McGowan and colleagues developed an evidence-

286 based guideline for Peer Review of Electronic Search Strategies (PRESS) for systematic reviews,

287 health technology assessments, and other evidence syntheses and recommended the main search

288 be conducted by a librarian and subsequently peer-reviewed by another librarian. ${ }^{25}$ It is essential to

289 keep clear and detailed documentation of the search strategy undertaken including search dates and

290 key terms used, sufficient to enable repetition of repeating searches (if required by other researchers).

291 Other additional sources such as hand searching of specific journals should be detailed including

292 journal names and years searched. If author contact for additional data was undertaken, it must be

293 stated in the review. The search for a scoping review may be quite iterative as reviewers become

294 more familiar with the evidence base, additional keywords and sources, and potentially useful search

295 terms may be discovered and incorporated into the search strategy. If this is the case, it is of the

296 utmost importance that the entire search strategy and results are transparent and auditable.

297 The language of sources of evidence that will be considered in the review must be prespecified in the

298 protocol. It is recommended that authors do not apply language restrictions to their protocols unless

299 there are reasonable justification such as feasibility or limitation of resources. 
302 Study selection must be prespecified in the protocol and based on the inclusion and exclusion criteria.

303 Study selection starts with review of both title and abstracts using the inclusion criteria followed by full

304 text retrieval of potentially relevant evidence for further review against the inclusion criteria. This

305 process is usually conducted by a minimum of two reviewers and any disagreements should be

306 resolved by either consensus or by a third reviewer. Description of the study selection process must

307 be presented in both a narrative and flow chart format as indicated in the PRISMA-ScR statement. ${ }^{16}$

308 Details of excluded sources at full text review must be appended to the review with reasons for their

309 exclusion. It is recommended that pilot testing of this process be undertaken by the review team to

310 ensure consistency of the approach taken in the study selection process.

311

312 Critical appraisal or risk of bias assessment is generally not recommended in scoping reviews as the

313 aim is to map the available evidence rather than provide a synthesized and clinically meaningful

314 answer to a question. Due to this, an assessment of methodological limitations or risk of bias of the

315 evidence included within a scoping review is generally not performed (unless there is a specific

316 requirement due to the nature of the scoping review aim). ${ }^{11,12,16}$

317

\section{Data extraction}

319 Data which is extracted from the evidence sources should align with the objectives and research

320 question of the scoping review. In scoping reviews, the data extraction process may be referred to as

321 'data charting,' although to be consistent with other evidence synthesis approaches, we have used

322 the term 'data extraction' in the updated guidance. A draft charting table or form should be developed

323 and piloted at the protocol stage to record the key information of the source, such as author,

324 reference, and results or findings relevant to the review question/s. This may be further refined at the

325 review stage and the charting table updated accordingly.

327 The scoping review protocol should include information about the potential data which could be

328 extracted from the included evidence sources to allow for transparency and clarity. The process of

329 data extraction should involve at least two reviewers to reduce the chance of errors and bias. Careful

330 record keeping should be kept either through a standardized form or table. The JBI offer an example

331 of a standardized data extraction form which can be utilized by all authors which can minimize

332 potential bias. ${ }^{26}$ However, these forms should be individualized to meet the needs of each scoping

333 review. It is recommended that the standardized data extraction form be piloted with two or more

334 members of the team on at least two to three studies prior to use to ensure that all necessary data will

335 be captured appropriately. Data extraction in scoping reviews can be an iterative process, often

336 requiring multiple refinements to be able to best meet the objectives and research question of the

337 scoping review. For example, an initial list of research characteristics may have been initially noted as

338 important i.e. year of research, location, or outcomes. However once reading several articles, authors

339 may want to list how those outcomes were measured to gain an in-depth understanding of how

340 researchers applied them and arrived at the subsequent results. 


\section{Data analysis}

344 An additional section in the updated guidance is a discussion on analyzing data in scoping reviews,

345 as this was highlighted as an area where additional information was required. ${ }^{15}$ Analysis of the data in 346 scoping reviews should be prespecified within the protocol to ensure transparency and justification of 347 the chosen approach. In most cases the intention of a scoping review is not to synthesize the results 348 or outcomes of the included sources. As such, for many scoping reviews, the analysis of the extracted 349 data should not involve anything more than basic descriptive analysis, i.e. frequency counts of 350 concepts, populations, or location of studies. These descriptive results can then be mapped in various 351 visual presentations, such as tables or graphs. The purpose of a scoping review and the type of data 352 that emerges in answer to the review question is not the type of evidence that lends itself to a meta353 analysis and little value would be gained in performing such an analysis. It is difficult to envisage a 354 case where further, in-depth quantitative analysis is required in scoping reviews, such as performing a 355 meta-analysis. Qualitative data should also be mostly descriptive and a synthesis utilizing a thematic 356 or meta-aggregative approach is not within the remit of a scoping review. Descriptive qualitative 357 techniques, such as basic coding of data to particular categories, may be a useful approach in some 358 scoping reviews, particularly when the purpose is to identify/clarify concepts or definitions within a 359 field or identifying key characteristics related to a concept. $21,27,28$ In summary, the way data is 360 extracted and analyzed in scoping reviews is largely dependent on the purpose of the review and 361 subject to the authors' judgement and creativity. The most important consideration regarding 362 extraction and analysis is that the authors are transparent and explicit in the approach they have 363 taken, including providing a rationale for their approach and clearly reporting extracted data and 364 analyses.

\section{Presentation of the results}

367 Data presentation approaches should be prespecified in the protocol stage. This could be further refined 368 in the review stage upon consideration of the contents of the included evidence. The results section of 369 a scoping review could be considered to contain two broad sections, the first of which describes the 370 results of the search strategy and selection process, including a PRISMA Flow diagram. The second 371 section will provide the key information or results relevant to the objectives/questions for the scoping review.

374 There are many options for presenting data in scoping reviews. The results of a scoping review may be presented as a map of the data extracted from the included papers in a diagrammatic or tabular form, and/or in a descriptive format that aligns with the objective/s and scope of the review. The elements of the PCC inclusion criteria may be useful to guide how the data should consider the best format(s) to present the results of the review for their audience. Presenting the results in a suitable and detailed format will allow the reviewers to identify gaps in the literature and map the available evidence. 
382 The update of the JBI scoping review methodology was driven by the need to provide further

383 clarification of when a scoping review is appropriate (and when it is not), how to extract, analyze and

384 present results, and to align with the development of the PRISMA-ScR. This article has provided an

385 overview of methods and up to date guidance for authors conducting scoping reviews that align with

386 the PRISMA-ScR to support reporting of scoping reviews. Further work to develop scoping review

387 methodology is planned by the JBI Scoping Review Methodology Group with current work focused on

388 producing guidance to appraise risk of bias (if required as an optional element of some reviews) in

389 scoping reviews; an article identifying key challenges and potential solutions to scoping reviews, and

390 the creation of a website to support dissemination and access to core scoping review methods. As

391 with all evidence synthesis methodologies, approaches to conducting and reporting scoping reviews

392 will be gradually enhanced and evolve in response to the needs of knowledge users as well as

393 through the experiences and familiarity of authors using current approaches. The JBI Scoping Review

394 Methodology Group is keen to continue providing authors with guidance and suggestions for

395 improving scoping review conduct and reporting and hopes that the latest iterations to the JBI

396 methodology are clear, helpful, and informative.

\section{References}

399

400

401

402

403

404

405

406

407

408

409

410

411

412

413

414

415

416

417

418

419

420

421

422

423

424

425

426

427

428

429

430

1. Bastian $\mathrm{H}$, Glasziou P, Chalmers I. Seventy-five trials and eleven systematic reviews a day: How will we ever keep up? PLoS Med. 2010; 7(9):e1000326.

2. Grant MJ, Booth A. A typology of reviews: an analysis of 14 review types and associated methodologies. Health Info Libr J. 2009; 26(2):91-108.

3. Tricco AC, Soobiah C, Antony J, Cogo E, MacDonald H, Lillie E, et al. A scoping review identifies multiple emerging knowledge synthesis methods, but few studies operationalize the method. J Clin Epidemiol. 2016; 73:19-28.

4. Sutton A, Clowes M, Preston L, Booth A. Meeting the review family: exploring review types and associated information retrieval requirements. Health Info Libr J. 2019; 36(3):202-22.

5. Ehrich K, Freeman GK, Richards SC, Robinson IC, Shepperd S. How to do a scoping exercise: continuity of care. Research, Policy and Planning. 2002; 20(1):25-9.

6. Anderson S, Allen P, Peckham S, Goodwin N. Asking the right questions: Scoping studies in the commissioning of research on the organisation and delivery of health services. Health Res Policy Syst. 2008; 6(1):7.

7. Tricco A, Lillie, E, Zarin, W, O'Brien, K, Colquhoun, H, Kastner, M, Levac, D, Ng, C, Pearson Sharpe, J, Wilson, K, Kenny, M, Warren, R, Wilson, C, Stelfox, HT \& Straus, SE. A scoping review on the conduct and reporting of scoping reviews. BMC Med Res Methodol. 2016; 16:15.

8. Pham M, Raji A, Greig J, Sargeant J, Papadopoulos A, McEwen S. A scoping review of scoping reviews: advancing the approach and enhancing the consistency. Res Synth Methods. 2014; 5(4):371-85.

9. Arksey H, O'Malley L. Scoping studies: towards a methodological framework. Int J Soc Res Methodol. 2005; 8(1):19-32.

10. Levac D, Colquhoun H, O'Brien KK. Scoping studies: advancing the methodology. Implement Sci. 2010; 5(1):69.

11. Khalil H, Peters M, Godfrey CM, Mclnerney P, Soares CB, Parker D. An evidence-based approach to scoping reviews. Worldviews Evid Based Nurs. 2016; 13(2):118-23.

12. Peters M, Godfrey C, Mclnerney P, Soares CB, Khalil H, Parker D. Chapter 11: Scoping reviews In: Aromataris E, Munn Z, editors. Joanna Briggs Institute Reviewer's Manual. The Joanna Briggs Institute; 2015.

13. Peters M, Godfrey C, Khalil H, Mclnerney P, Parker D, Soares C. Guidance for conducting systematic scoping reviews. Int J Evid Based Healthc. 2015; 13(3):141-6. 
14. Peters M, Godfrey C, Mclnerney P, Soares CB, Khalil H, Parker D. Chapter 11: Scoping reviews. In: Aromataris E, Munn Z, editors. Joanna Briggs Institute Reviewer's Manual. The Joanna Briggs Institute; 2017. 15. Khalil H, Bennett M, Godfrey C, Mclnerney P, Munn Z, Peters M. Evaluation of the JBI scoping reviews methodology by current users. Int J Evid Based Healthc. 2020; 18(1):95-100. 16. Tricco AC, Lillie E, Zarin W, O'Brien KK, Colquhoun H, Levac D, et al. PRISMA extension for scoping reviews (PRISMA-ScR): checklist and explanation. Ann Intern Med. 2018; 169(7):467-73. 17. Peters M, Godfrey C, Mclnerney P, Munn Z, Tricco A, Khalil H. Chapter 11: Scoping reviews. In: Aromataris E, Munn Z, editors. Joanna Briggs Institute Reviewer's Manual. The Joanna Briggs Institute; 2020.

441 18. Grimshaw J. A knowledge synthesis chapter. Canadian Institutes

442 of Health Research; 2010. 19. Tricco AC, Lillie E, Zarin W, O'Brien K, Colquhoun H, Kastner M, et al. A scoping review on the conduct and reporting of scoping reviews. BMC Med Res Methodol. 2016; 16:15. 20. Munn Z, Peters MDJ, Stern C, Tufanaru C, McArthur A, Aromataris E. Systematic review or scoping review? Guidance for authors when choosing between a systematic or scoping review approach. BMC Med Res Methodol. 2018; 18(1):143. 21. Harfield SG, Davy C, McArthur A, Munn Z, Brown A, Brown N. Characteristics of Indigenous primary health care service delivery models: a systematic scoping review. Global Health. 2018; 14(1):12.

22. Watson R, Parr J, Joyce C, May C, Le Couteur A. Models of transitional care for young people with complex health needs: a scoping review. Child Care Health Dev. 2011; 37(6):780-91. 23. Tricco A, Zarin W, Ghassemi M, Nincic V, Lillie E, Page M, et al. Same family, different species: methodological conduct and quality varies according to purpose for five types of knowledge synthesis. J Clin Epidemiol. 2018; 96:133-42.

456

24. Tricco A. What Review is Right for You? : Knowledge Translation Program; 2020 [cited 20205 May]. Available from: https://whatreviewisrightforyou.knowledgetranslation.net/ 25. McGowan J, Sampson M, Salzwedel D, Cogo E, Foerster V, Lefebvre C. PRESS peer review of electronic search strategies: 2015 guideline statement. J Clin Epidemiol. 2016; 75:40-6. 26. Peters M, Godfrey C, Mclnerney P, Munn Z, Tricco A, Khalil H. Appendix $11.1 \mathrm{JBI}$ template source of evidence details, characteristics and results extraction instrument. In: Aromataris E, Z M, editors. Joanna Briggs Institute Reviewer's Manual. JBI; 2020. 27. Davy C, Harfield S, McArthur A, Munn Z, Brown A. Access to primary health care services for Indigenous peoples: a framework synthesis. Int J Equity Health. 2016; 15(1):163. implementation in rehabilitation: a scoping review. PM R. 2018; 10(11):1237-51.

29. Munn Z, Stern C, Aromataris E, Lockwood C, Jordan Z. What kind of systematic review should I conduct? A proposed typology and guidance for systematic reviewers in the medical and health sciences. BMC Med Res Methodol. 2018; 18(1):5.

470 30. Snilstveit B, Vojtkova M, Bhavsar A, Stevenson J, Gaarder M. Evidence \& gap maps: A tool for 